\title{
Éditorial. Donner à lire les humanités numériques francophones (2)
}

Editorial. Francophone Digital Humanities to Read (2)

\section{(2) OpenEdition}

\section{Journals}

Édition électronique

URL : https://journals.openedition.org/revuehn/507

DOI : $10.4000 /$ revuehn.507

ISSN : 2736-2337

Éditeur

Humanistica

Référence électronique

"Éditorial. Donner à lire les humanités numériques francophones (2) », Humanités numériques [En

ligne], 2 | 2020, mis en ligne le 01 juin 2020, consulté le 16 juillet 2021. URL : http://

journals.openedition.org/revuehn/507 ; DOI : https://doi.org/10.4000/revuehn.507

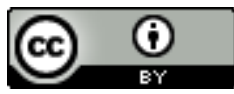

Les contenus de la revue Humanités numériques sont mis à disposition selon les termes de la Licence Creative Commons Attribution 4.0 International. 


\section{humanités \\ numériques}

\section{Éditorial. Donner à lire les humanités numériques francophones (2) \\ Editorial. Francophone Digital Humanities to Read (2)}

\section{Entrées d'index}

MOTS-CLÉS : humanités numériques

KEYWORDS: digital humanities

Un texte introductif peut se donner pour ambition de placer les articles recueillis dans une perspective particulière, parfois en les résumant. Si nous en croyons notre expérience de lecteurs, cette formule traditionnelle est souvent peu stimulante. Nous aimerions plutôt que les éditoriaux de la revue soient les traces d'un moment dans la vie d'une communauté, de ce qui fait bruisser les réseaux sociaux et anime les séminaires, voire de ce qui tisse ou transforme la toile de fond des travaux savants.

\section{Sortir du confinement}

Notre intention initiale était de publier les numéros 1 et 2 d'Humanités numériques au moment du premier colloque d'Humanistica, qui devait se tenir à Bordeaux au mois de mai 2020. La crise sanitaire du Covid-19 a entraîné l'annulation de cet événement ; une page d'" Archives du colloque " recense les communications acceptées et les liens vers les matériaux mis à disposition. Profitons de cet éditorial pour lancer une invitation spéciale : que celles et ceux qui auraient contribué au colloque n'hésitent pas à soumettre des propositions d'articles à la revue. 
Peut-être certains textes reflèteront-ils bientôt cette période de confinement où les usages numériques ont fait l'objet d'une réflexion, parfois d'une prise de conscience, dans tous les milieux professionnels, et en particulier dans le monde de l'enseignement ? Souvent situées à l'épicentre des expérimentations médiatiques, les humanités numériques auront sans doute quelque chose à dire de notre expérience partagée.

\section{Deux varia pour commencer}

Pour ouvrir la revue, notre premier appel proposait des thématiques " Disciplines et/ou humanités numériques », « Regards sur des projets en humanités numériques »- qui n’ont pas suscité suffisamment d'articles pour constituer des ensembles cohérents. Sans doute ces sujets reviendront-ils, car ils sont importants. Ce premier appel a cependant été un succès, puisque nous avons reçu plus de 50 propositions. Outre le comité de direction, plus de 75 collègues ont participé au processus d'évaluation, que nous avons voulu bienveillant et constructif. Ce sont des versions révisées que vous lirez, dans la plupart des cas, tandis que les auteurs de la majorité des articles qui n'ont pas été acceptés ont été incités à soumettre un texte refondu sur la base des conseils reçus.

Les numéros 1 et 2 sont donc des varia, selon la dénomination en usage sur OpenEdition Journals. Nous nous sommes gardés d'introduire a posteriori des rubriques artificielles ou d'imposer un classement chronologique, bien que nous soyons très heureux de constater une vaste amplitude temporelle. Il nous a semblé préférable d'identifier de grandes orientations, sur lesquelles nous avons fondé le détail des sommaires, d'une manière organique. C'est ainsi que nous avons abouti pour l'instant aux pôles thématiques suivants : histoire(s) des humanités numériques ; édition et corpus, de l'Antiquité au $\mathrm{XxI}^{\mathrm{e}}$ siècle ; théories, méthodes et controverses ; retours d'expérience. Il sera intéressant de voir si la pertinence de ces catégories se confirme au fil des années. De toute évidence, un tel découpage implicite est loin de rendre justice aux multiples dimensions des humanités numériques. Donnons ici quelques statistiques qui nous semblent éclairantes.

Il est possible d'esquisser, d'ores et déjà, une typologie des auteurs selon leur sexe, leur pays d'activité, leur situation professionnelle et les domaines ou disciplines dans lesquels ils exercent. Les 19 articles ont été produits par 45 auteurs, 24 hommes et 21 femmes, sans qu'il ait fallu faire de la parité un critère. Ces contributeurs résident dans trois pays : 16 articles nous parviennent de France, deux du Canada et un de Suisse. Une telle répartition n'est guère surprenante, car il s'agit de proportions assez semblables à celles des membres d'Humanistica ; nous espérons néanmoins que les cultures francophones représentées seront plus nombreuses à l'avenir.

Les différences entre pays et entre institutions rendent parfois délicate l'appréciation de l'insertion professionnelle des auteurs. On peut néanmoins remarquer que, d'après notre estimation, $80 \%$ de ces collègues occupent des postes permanents, les $20 \%$ restants ayant actuellement une situation de chercheurs post-doctorants. Ce dernier pourcentage paraît élevé. Il est bien sûr caractéristique de la relative jeunesse de la communauté des humanités numériques. Il témoigne aussi de la part grandissante de l'emploi temporaire dans la recherche en gé- 
néral et, en particulier, dans les projets à forte composante numérique. Il s'agit, là encore, d'une question que, sur la durée, notre projet éditorial peut contribuer à documenter et analyser. Parmi les permanents, les chercheurs ou enseignants-chercheurs représentent environ les deux tiers des effectifs et ont à parts à peu près égales des postes de rang A et de rang $B$, pour reprendre une division française courante ; un tiers des auteurs a le statut d'ingénieur, soit en informatique, soit en documentation et en édition.

En ce qui concerne la taille des articles, nous avions décidé d'exploiter la grande liberté permise par l'édition électronique, tout en indiquant 50 ooo signes comme une limite courante. Notes et espaces comprises, la moyenne des articles est de 55000 signes, les deux tiers des articles se situent entre 50 ooo et 60 ooo environ, le plus court comprenant 30000 signes et le plus long, 77 ooo signes.

La forme des textes s'adapte au propos, du discours argumentatif ne passant que par les mots jusqu'au commentaire richement illustré de ressources en ligne. Les captures d'écran sont nombreuses, tout comme les diagrammes, les graphiques et les extraits de code ; dans ce dernier cas, il est également possible d'intégrer sur la plateforme des fragments de divers langages informatiques dans une présentation efficace, en recourant à la coloration syntaxique. Nous n'avons pas encore reçu de data paper, mais une autrice renvoie à un site publiant les données qu'elle exploite et des visualisations interactives.

Pour permettre des parcours riches et une analyse plus fine du corpus des articles, nous avons harmonisé et enrichi les mots-clés fournis par les auteurs. Nous étiquetons ainsi les disciplines à partir d'un référentiel constitué pour la revue, fondé principalement sur l'index du catalogue d'OpenEdition et sur la liste des sciences humaines et sociales du thésaurus du projet Savoirs. Nous ajoutons également des termes désignant les objets, activités, techniques et technologies des humanités numériques, en nous inspirant de la taxinomie TaDiRAH, qui a l'avantage d'avoir été développée spécifiquement pour le domaine.

\section{À suivre : un numéro thématique}

Le prochain numéro, le troisième, sera thématique, conformément à l'alternance que nous souhaitons entre numéros composés d'articles variés et numéros consacrés à un sujet ou un domaine. Il portera sur les " humanités numériques spatialisées ": comme le précisait l'appel, il s'agit de rendre compte de diverses approches spatiales permises par les systèmes d'information géographique et géohistoriques. Nous avons reçu une vingtaine de propositions, dont les collègues invités à diriger ce numéro coordonnent actuellement l'évaluation. 


\section{Commentez, critiquez, contribuez!}

Lieu de publication, la revue a également vocation à être un espace de débat ou une caisse de résonance. Les sujets des textes sont bien sûr un reflet indirect des questions pratiques ou théoriques du moment. Les numéros thématiques ont également cette fonction, et nous vous encourageons à en proposer.

D'autres formes de dialogue peuvent néanmoins être inventées : on peut imaginer que des articles, individuels ou collectifs, approfondissent une discussion née sur la liste de diffusion Digital Humanities ou sur les réseaux sociaux, par exemple, ou bien réagissent à un article antérieur. Nous sommes ouverts à de nouvelles rubriques ou à de nouveaux modèles d'article scientifique, avec un critère clair : qu'ils alimentent la conversation critique au sujet des humanités numériques.

Nous vous souhaitons autant de plaisir et d'intérêt à lire ces deux premiers numéros que nous en avons eu à les préparer.

Le comité de direction : Aurélien Berra, Emmanuel Château-Dutier, Sébastien Poublanc, Émilien Ruiz, Nicolas Thély (membres actuels), Emmanuelle Morlock (membre durant la préparation de ces numéros, jusqu'en octobre 2019)

\section{Droits d'auteur}

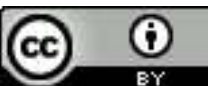

Les contenus de la revue Humanités numériques sont mis à disposition selon les termes de la Licence Creative Commons Attribution 4.0 International. 